Proceedings

\title{
Watershed Prioritization of Upper Kosi Watershed Based on Soil Erodibility Computed Through TanDEM-X DEM and TOPSIS-AHP Ensemble Model ${ }^{\dagger}$
}

\author{
Brij Mohan Bohra (D) and Ashutosh Bhardwaj *(D) \\ Indian Institute of Remote Sensing, Dehradun 248001, India; b2bohra1995@gmail.com \\ * Correspondence: ashutosh@iirs.gov.in \\ + Presented at the 1st International Electronic Conference on Applied Sciences, \\ 10-30 November 2020; Available online: https:/ / asec2020.sciforum.net/.
}

Published: 10 November 2020

\begin{abstract}
In recent times, soil erosion has become one of the major environmental issues considering large scale anthropogenic activities and frequent extreme weather events globally. These activities and events cause the wearing away of top soil, initiating loss in agricultural land, and triggering disasters like landslides in the hilly regions. So, such regions demand soil conservation measures such as plantation, and construction of check dams. Due to limited resources in developing countries like India, prioritization of land-based on susceptibility to soil erosion makes the implementation of these measures efficient. In the present study, the openly accessible TanDEM-X $90 \mathrm{~m}$ DEM and TOPSIS-AHP ensemble model are used for prioritization of Upper Kosi Watershed based on soil erodibility. This watershed having fifth (5th) order perennial Kosi river is a showcase for different landscapes, biodiversity, micro-climate, and hydrogeology. TanDEM-X DEM is preprocessed for stream network generation and catchment delineation which is further used for extraction of 10 morphometric attributes (MAs) of each sub-watershed. AHP model is used for calculating the weights of MAs. AHP model concludes that Watershed slope, Stream frequency, and Drainage density play a greater role in soil erosion as compared to other MAs. Morphometric parameters and its weightage is utilized in the execution of the TOPSIS model to calculate the closest coefficient for each sub-watershed. Further, these closest coefficient is classified into low (0.147-0.167), medium (0.167-0.326), and high (0.326-0.794) susceptible zones for prioritization of sub-watershed based on the geometric mean method. This study highlights that Upper Kosi watershed contains $4.73 \%$, $38.79 \%, 56.48 \%$ of its area in high, medium, and low susceptible zone respectively. The result can assist the decision-makers and planners in choosing suitable regions depending on the available resources for conservation measures in Upper Kosi watershed.
\end{abstract}

Keywords: digital elevation model; soil erodibility; watershed prioritization; catchment; morphometric attributes

\section{Introduction}

Soil is one of the most valuable natural resources for the proper functioning of an ecosystem. It contributes in providing nutrients to plants and habitat for organic process, filtering water, managing solid waste etc [1]. It degrades naturally with time due to water and wind especially in temporal region like India. This natural process is known as Soil erosion [2]. Pace of this process has been increased exponentially in recent decades due to anthropogenic activities such as deforestation, agricultural intensification, urbanization and land reclamation etc. It causes loss of 
agricultural land and natural disaster like landslides [3]. It requires soil conservation measures such as plantation, step farming in hilly area and construction of ponds, check dams to reduce the impact of this problem [4]. Drainage basin which transports the eroded soil through water to a common outlet can be considered as a unit by policy-makers and planners to execute the soil conservation measures [5].

Drainage basin can be quantified by some attributes based on linear, areal and relief aspect. These attributes are known as Morphometric attributes. Remote sensing and GIS is a simplest tool to determine the morphometry of a basin. It only requires a well accurate digital elevation model as an input to delineate the catchment and its stream network [6].

Most of the major catchment has its area in hundreds of square kilometers. It is not possible for any developing country like India to implement soil conservation measures for such a large basin in single time. Therefore, it requires the prioritization of watershed based on its susceptibility to soil erosion. There are various model or numerical techniques such as weighted sum analysis (WSA) [7], soil and water assessment tools (SWAT) [8], sediment yield index (SYI) [9] for prioritization of catchment on the basis of soil erodibility. However, in recent time, mathematical models such as AHP [10], Fuzzy-AHP [11], TOPSIS [12], TOPSIS-AHP are widely used multi-criteria decision making techniques for ranking of watershed based on soil erodibility. These MCDM techniques are suitable for basin with limited data.

This study uses TanDEM-X 90m DEM and TOPSIS-AHP ensemble model to classified the Upper Kosi watershed in different susceptible zones for soil erodibility. This study starts with the computation of morphometric attributes for watershed and then it is followed by catchment prioritization.

\section{Study Area}

The Upper Kosi watershed shown in Figure 1 in Kumaun region was selected for soil erodibility prioritization because of 5th order perennial Kosi river. It is a showcase of different landscape (tectonically controlled and fluvial), landform (dissected valley, terraces, ridge, and streams), biodiversity (floral and faunal), micro-climate, habitat, demography and hydrogeology (soil, geology and water) that falls in Almora district of Uttarakhand, India. The Kosi river originates from its north most point at Pinath (near Kausani, district Almora), which flows downward and join Ramganaga in Ramnagar as its major tributary. Geographically, the catchment of Upper Kosi River has its spatial extension between $29^{\circ} 37^{\prime} 30^{\prime \prime}$ to $29^{\circ} 52^{\prime} 20^{\prime \prime} \mathrm{N}$ and $79^{\circ} 31^{\prime} 00^{\prime \prime}$ to $79^{\circ} 51^{\prime} 45.10^{\prime \prime} \mathrm{E}$ which covers an area of about $462 \mathrm{sq}$. Km. The absolute relief of the catchment ranges between $1106 \mathrm{~m}$ and $2758 \mathrm{~m}$ above mean sea level and represents wide range of elevation in the Kumaun Himalayan [13].

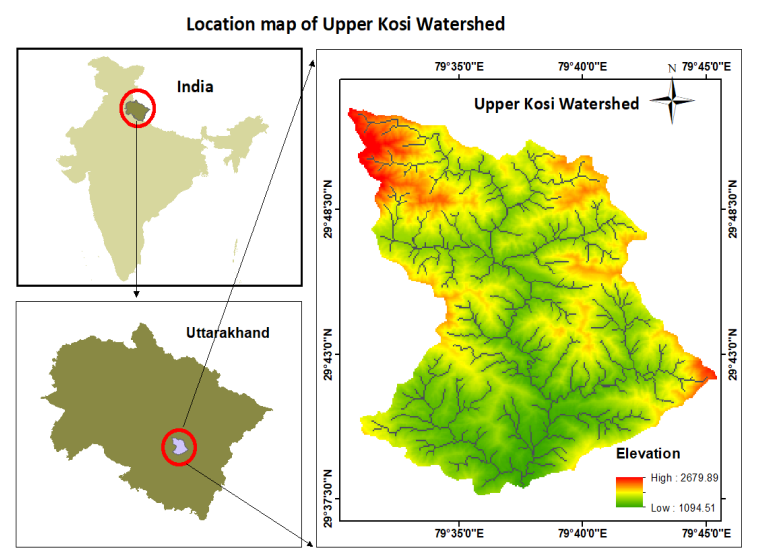

Figure 1. Upper Kosi Watershed with stream map. 


\section{Material and Method}

\subsection{Dataset Used}

TanDEM-X $90 \mathrm{~m}$ DEM is utilized to delineate the catchment. It is a product of TanDEM-X earth observation radar mission. It has been generated from a bi-static SAR technique that consist of SAR interferometer built by two satellites flying in separation of $120 \mathrm{~m}$ to $500 \mathrm{~m}$. It has a pixel spacing of 3 arcseconds with absolute height error of magnitude below than $10 \mathrm{~m}$ [14]. This freely available product is downloaded from the portal of EOC Geoservice of Earth Observation Center (EOC) of the German Aerospace Center(DLR).

\subsection{Morphometric Analysis}

In this study, sub-watersheds are basic hydrological units to calculate the morphometric attributes. For that, DEM attempted to delineate the boundaries of sub-watershed using watershed analysis tools of TNTmips 2019 software [15]. Steps such as fill sinking of DEM, flow direction and flow accumulation were performed as preprocessing to generate the stream network. These all preprocessing were performed in Arc Hydro Tools of ArcGIS 10.4. Generated stream network is analyzed in spatial analyst tool to determine the stream order and stream length. This stream network map provides some basic morphometric parameter such as area, perimeter, stream order, stream length, stream number and elevation [16]. However, other parameters were determined by table of formulae given in Sadhasivam et al. (2020) [15]. In the present study, 10 morphometric parameters such as Drainage density (Dd), Stream frequency $(\mathrm{Fu})$, Watershed slope (Sw), Length of overland flow (Lo), Relative relief $(\mathrm{Rr})$, Ruggedness number $(\mathrm{Rn})$, Mean bifurcation ratio $(\mathrm{Rbm})$, Texture ratio $(\mathrm{T})$, Elongation ration (Rc), Infiltration rate (If) were selected based on its sensitivity to soil erosion to prioritize the sub-watershed [7].

\subsection{Multi Criteria Decision Making Model}

MCDM methods are being used since 1960s. It is widely accepted and commonly used by decision makers to solve area specific problem such as safety and risk management, energy , strategic management etc. [17] . It is basically divided into two types- Direct MCDM method and Indirect MCDM method. Direct method involves the weightage of criteria based on survey while indirect method is based on the previous studies and experience of decision makers [18] . In this study, indirect method is used wherein relative weightage is selected for the 10 morphometric attributes using AHP (Analytical Hierarchical Process) technique. After that, TOPSIS method is used for ranking the sub-watershed in inference to soil erodibility.

\subsubsection{Analytical Hierarchical Process Model (AHP)}

It is a mathematical model introduced by Thomas Saaty in 1977. In the model, selected problem statement is structured in hierarchical order descending from an objective to criterion and then alternative in succession [19]. In AHP, pairwise comparison matrix (PWC) A is generated in which importance of each criteria is assigned between 1 to 9 against each other [20].

$$
A=\left[\begin{array}{cccccc}
a_{11} & a_{12} & \ldots & a_{1 k} & \ldots & a_{1 n} \\
a_{21} & a_{22} & \ldots & a_{2 k} & \ldots & a_{2 m} \\
\vdots & \vdots & \vdots & \vdots & \vdots & \\
a_{j 1} & a_{j 2} & \ldots & a_{j k} & \ldots & a_{j m} \\
\vdots & \vdots & \vdots & \vdots & \vdots & \\
a_{m 1} & a_{m 2} & \ldots & a_{m k} & \ldots & a_{m m}
\end{array}\right]
$$


where $a_{j k}=$ importance of $\mathrm{j}$-th criteria relative to the i-the criteria. To check the consistency of PWC martix, Consistency ratio $(C R)$ is calculated using the following formula:

$$
C R=\frac{C I}{R I}
$$

where $C I=$ consistency index.

$C I$ is computed as follows:

$$
C I=\frac{\lambda_{\max }-m}{m-1}
$$

$\lambda_{\max }=$ maximum eigen values of PWC matrix.

$m=$ number of criteria.

CR should be less than $10 \%$ to declare the model consistent. If model is consistent then acquired weightage of each MAs is used in TOPSIS model.

\subsubsection{TOPSIS}

TOPSIS stands for the 'Technique for Order of Preference by Similarity to Ideal Solution'. It is developed by Ching-Lai Hwang and Yoon in 1981. It works on the principle of 'Eculidean distance approach' i.e., alternative having closest to the positive ideal solution (PIS) and farthest to the negative ideal solution (NIS) is preferred [21] . The following equations are used in sequence to execute this model:

1. Preparation of decision matrix $P$ using $m$ choices and $n$ parameters.

$$
P=\left[\begin{array}{ccccc}
p_{11} & p_{12} & p_{13} & \ldots & p_{1 n} \\
p_{21} & p_{22} & p_{23} & \ldots & p_{2 n} \\
\vdots & \vdots & \vdots & \vdots & \vdots \\
p_{m 1} & p_{m 2} & p_{m k} & \ldots & p_{m n}
\end{array}\right]
$$

where $p_{m n}=$ value of parameter $n$ for choice $m$

2. Calculation of normalized decision matrix using formula for its each element.

$$
r_{\mathrm{ij}}=\frac{p_{\mathrm{ij}}}{\sqrt{\sum_{i=1}^{m} p_{\mathrm{ij}}^{2}}}
$$

where $r_{\mathrm{ij}}=$ element of normalized matrix having parameter $j$ of choice $i$

3. Construction of weighted normalized matrix.

$$
u_{\mathrm{ij}}=r_{\mathrm{ij}} * w_{\mathrm{j}}
$$

where $u_{\mathrm{ij}}=$ elements of weighted normalized matrix having parameter $j$ of choice $i, w_{j}=$ weight of each criteria calculated from AHP

4. Estimations of positive $\left(P^{+}\right)$and negative $\left(P^{-}\right)$ideal solution;

$$
\begin{aligned}
P^{+} & =\left\{\left(\operatorname{maxu}_{\mathrm{ij}} \mid j \in I\right),\left(\operatorname{minu}_{\mathrm{ij}} \mid j \in I^{\prime}\right) \mid i=1, \ldots, n\right\}=\left\{u_{1}{ }^{+}, u_{2}{ }^{+}, \ldots, u_{\mathrm{n}}{ }^{+}\right\} \\
P^{-} & =\left\{\left(\operatorname{minu}_{\mathrm{ij}} \mid j \in I\right),\left(\operatorname{maxu}_{\mathrm{ij}} \mid j \in I^{\prime}\right) \mid i=1, \ldots, n\right\}=\left\{u_{1}{ }^{-}, u_{2}{ }^{-}, \ldots, u_{\mathrm{n}}{ }^{-}\right\}
\end{aligned}
$$

where $I$ and $I^{\prime}$ related to the PIS and NIS respectively and defined through literature review. 
5. Measurement of Euclidean distance from PIS and NIS:

$$
\begin{gathered}
S_{i}^{+}=\sqrt{\sum_{j=1}^{n}\left(u_{i j}-u_{i}^{+}\right)^{2}}, \quad i=1, \ldots, n \\
S_{i}^{-}=\sqrt{\sum_{j=1}^{n}\left(u_{i j}-u_{i}^{-}\right)^{2}}, \quad i=1, \ldots, n
\end{gathered}
$$

where $S_{i}{ }^{+}$and $S_{i}^{-}$represents euclidean distance from PIS and NIS.

6. Calculation of Closest coefficient $\left(\mathrm{C}_{\mathrm{i}}^{+}\right)$:

$$
C_{\mathrm{i}}^{+}=\frac{S_{i}^{+}}{S_{i}^{+}+S_{i}^{-}}
$$

Value of closest coefficient varies between 0 and 1 . The alternative which has the largest closest coefficient is given highest priority and vice versa.

\section{Results and Discussion}

\subsection{Morphometric Analysis of Watershed}

TanDEM-X $90 \mathrm{~m}$ is selected for the morphometric analysis due to its accuracy better than $0.53 \mathrm{~m}$ [22] . For this analysis, Upper Kosi watershed in Uttarakhand is chosen because of perenniality of Kosi river and adequate rainfall in the watershed which shows dendritic and sub-dendritic drainage pattern in stream map (Figure 1). Methodology as flow chart shown in Figure 2 is used for the analysis. 21 subwatersheds are generated for the region and 10 morphometric attributes are calculated for the each sub-watershed shown in Table 1. Dd is highest for sub-watershed fid 17 which means the least permeability for sub-watershed. Highest value of Fu for sub-watershed fid 21 shows the more runoff comparable to others. Lo ranges from 0.35 (WS fid17) to 0.57 (WS fid14) for the waterhsed which describes quicker runoff process for sub-watershed fid17 and slower for sub-watershed fid14 theoretically. Rbm value does n't come under 3 to 5 for any sub-watershed which also describes that geological structure of sub-watershed will play an important role in disturbing sub-watershed [7]. $\mathrm{T}$ has positive relation with soil erosion which is highest for sub-watershed fid4 (1.799). 'If' values range from 1.03 to 5.64 which means high surface runoff in watershed, specifically for sub-watershed fid 21. Sw has a direct correlation with the erosion showing highest degree of erosion for sub-watershed fid 21 (323.77). Sw value for all sub-watershed is too high (27.73-323.77) due to high basin elevation with respect to its area. This is an indication of high flood peak at the time of rainfall. Rr shows variability from 22.81 (WS fid11) to 58.17 (WS fid2) indicating highest erosion prone sub-watershed fid2 due to increasing montonicity of $\mathrm{Rr}$ with soil erosion. Rn has positive correlation with erosion which ranges from 0.17 (WS fid21) to 0.96 (WS fid2) for the watershed. Rc which is a shape parameter has negative relation with soil erosion. Its value ranges from 0.27 (WS fid11) to 0.44 (WS fid7) [7]. 


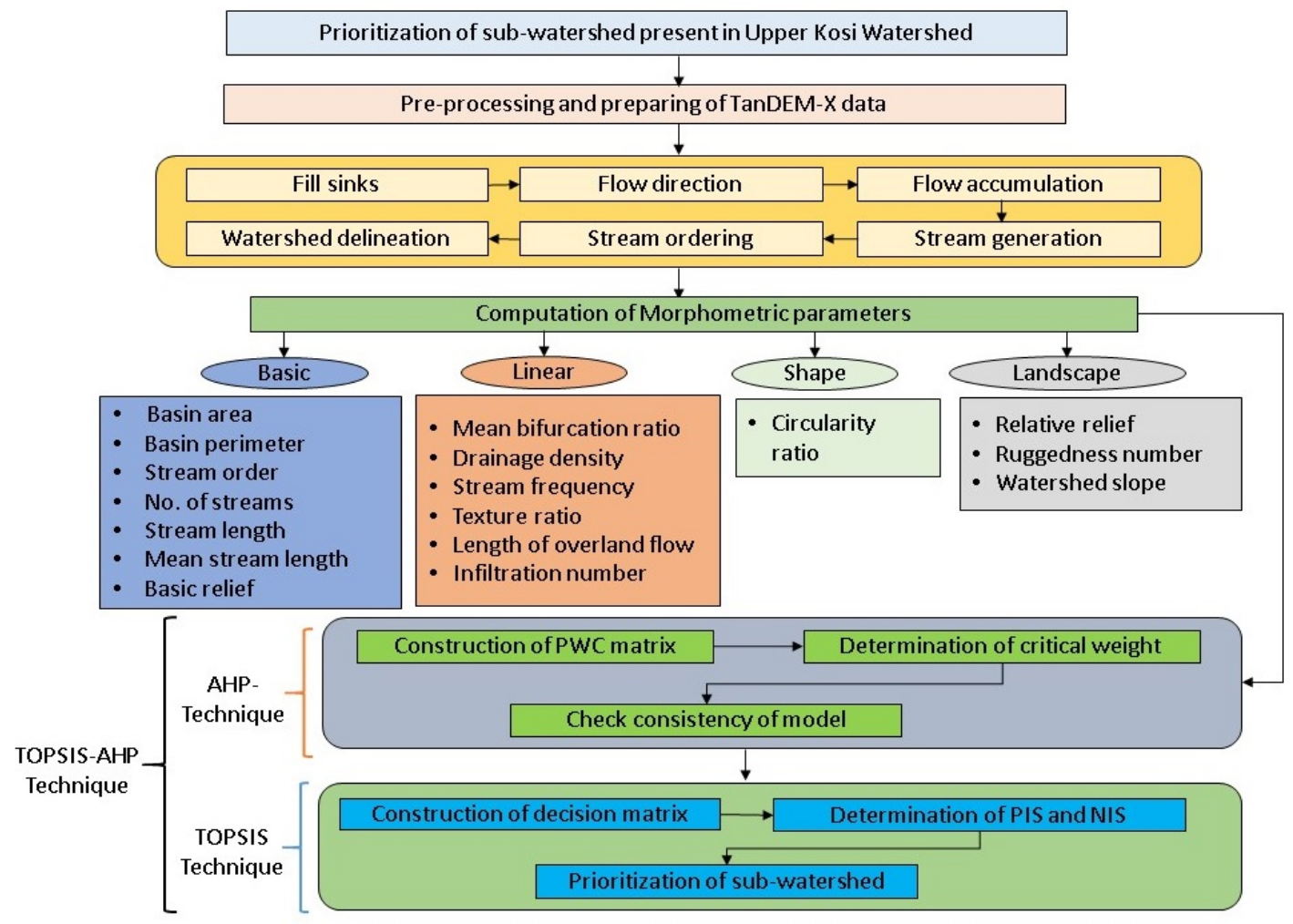

Figure 2. Flow chart for soil erodibility prioritization.

Table 1. Computed morphometric attributes of sub-watershed.

\begin{tabular}{ccccccccccc}
\hline SW & Dd & Fu & Lo & Rbm & T & If & Sw & Rr & Rn & Rc \\
\hline fid0 & 1.004 & 1.676 & 0.498 & 2.018 & 1.349 & 1.683 & 41.545 & 33.457 & 0.815 & 0.414 \\
fid1 & 0.99 & 1.046 & 0.505 & 1.143 & 0.643 & 1.035 & 76.53 & 47.074 & 0.909 & 0.331 \\
fid2 & 0.938 & 1.288 & 0.533 & 1.875 & 0.691 & 1.207 & 108.347 & 58.174 & 0.967 & 0.359 \\
fid3 & 1.036 & 1.095 & 0.482 & 1.583 & 0.837 & 1.135 & 32.574 & 24.897 & 0.718 & 0.321 \\
fid4 & 1.059 & 1.668 & 0.472 & 1.599 & 1.799 & 1.766 & 31.191 & 33.65 & 0.948 & 0.375 \\
fid5 & 1.015 & 1.319 & 0.493 & 2.092 & 1.272 & 1.338 & 22.817 & 22.014 & 0.767 & 0.343 \\
fid6 & 1.005 & 1.454 & 0.498 & 1.417 & 0.731 & 1.461 & 72.498 & 36.451 & 0.645 & 0.355 \\
fid7 & 1.135 & 2.65 & 0.441 & 1.567 & 1.103 & 3.007 & 121.24 & 50.486 & 0.524 & 0.444 \\
fid8 & 0.963 & 1.272 & 0.519 & 2.042 & 1.041 & 1.225 & 30.646 & 25.08 & 0.825 & 0.324 \\
fid9 & 1.043 & 1.489 & 0.479 & 1.571 & 1.158 & 1.553 & 35.48 & 27.587 & 0.708 & 0.365 \\
fid10 & 0.935 & 1.27 & 0.535 & 1.143 & 0.809 & 1.188 & 71.339 & 45.441 & 0.901 & 0.431 \\
fid11 & 1.148 & 1.079 & 0.436 & 1.317 & 0.825 & 1.238 & 28.976 & 22.147 & 0.679 & 0.273 \\
fid12 & 1.128 & 1.196 & 0.443 & 1.917 & 0.906 & 1.349 & 45.267 & 34.3 & 0.705 & 0.411 \\
fid13 & 1.057 & 1.239 & 0.473 & 1.733 & 0.977 & 1.31 & 29.096 & 22.933 & 0.644 & 0.333 \\
fid14 & 0.871 & 1.518 & 0.574 & 2.25 & 0.955 & 1.322 & 51.427 & 32.333 & 0.661 & 0.443 \\
fid15 & 0.932 & 1.489 & 0.537 & 1.675 & 1.152 & 1.387 & 57.002 & 44.117 & 0.91 & 0.415 \\
fid16 & 1.071 & 1.375 & 0.467 & 2.811 & 1.114 & 1.473 & 35.142 & 28.47 & 0.739 & 0.366 \\
fid17 & 1.41 & 2.431 & 0.355 & 0.667 & 0.545 & 3.429 & 195.468 & 43.775 & 0.285 & 0.306 \\
fid18 & 0.961 & 1.258 & 0.52 & 1.6 & 0.852 & 1.209 & 46.151 & 31.27 & 0.801 & 0.345 \\
fid19 & 0.986 & 1.474 & 0.507 & 1.681 & 1.246 & 1.453 & 27.731 & 23.442 & 0.668 & 0.378 \\
fid21 & 1.375 & 4.107 & 0.364 & 0.5 & 0.65 & 5.645 & 323.775 & 51.256 & 0.172 & 0.431 \\
\hline
\end{tabular}

\subsection{Prioritization Based on TOPSIS-AHP Model}

In parallel to morphometric analysis, pairwise comparison matrix is generated for these attributes using literature reviews. Element value of PWC matrix (Table 2) shows the importance of MA in row with respect to its corresponding MA in column where value, greater than 1 means MA in row is more important than MA in column. After that, consistency ratio is calculated using the Equation (1) which 
is below than 0.1. It shows that AHP model is consistent. Computed weightage of each MAs shown in Table 3 is used in TOPSIS model. Table 3 obtained from AHP model summarizes that soil erodibility largely depends on $\mathrm{Sw}, \mathrm{Fu}$ and $\mathrm{Dd}$. It also concludes that there is very less impact of If, Rc, Lo and $\mathrm{Rbm}$ in process of soil erodibility. The output of AHP model shows the consistency with the results of Auoragh (2018) [12] and Amiri et al. (2019) [23].

Table 2. Elements of PWC matrix.

\begin{tabular}{ccccccccccc}
\hline & Dd & Fu & Sw & Lo & Rr & Rn & Rbm & T & Rc & If \\
\hline Dd & 1 & 2 & 0.7 & 2 & 0.6 & 0.4 & 4 & 3 & 7 & 7 \\
$\mathrm{Fu}$ & 0.5 & 1 & 0.5 & 2 & 4 & 4 & 2 & 2 & 5 & 4 \\
$\mathrm{Sw}$ & 1.4 & 2 & 1 & 5 & 3 & 3 & 4 & 4 & 5 & 5 \\
$\mathrm{Lo}$ & 0.5 & 0.5 & 0.2 & 1 & 0.5 & 0.5 & 0.7 & 2 & 5 & 7 \\
$\mathrm{Rr}$ & 1.7 & 0.3 & 0.3 & 2 & 1 & 2 & 2 & 3 & 7 & 4 \\
$\mathrm{Rn}$ & 2.5 & 0.3 & 0.3 & 2 & 0.5 & 1 & 2 & 3 & 4 & 5 \\
$\mathrm{Rbm}$ & 0.3 & 0.5 & 0.3 & 1.43 & 0.5 & 0.5 & 1 & 0.7 & 7 & 7 \\
$\mathrm{~T}$ & 0.3 & 0.5 & 0.3 & 0.5 & 0.3 & 0.3 & 1.4 & 1 & 4 & 4 \\
$\mathrm{Rc}$ & 0.1 & 0.2 & 0.2 & 0.2 & 0.1 & 0.3 & 0.1 & 0.3 & 1 & 2 \\
$\mathrm{If}$ & 0.1 & 0.3 & 0.2 & 0.14 & 0.3 & 0.2 & 0.1 & 0.3 & 0.5 & 1 \\
\hline
\end{tabular}

Table 3. Computed weight of each MA using AHP model.

\begin{tabular}{cccccccccc}
\hline Dd & Fu & Sw & Lo & Rr & Rn & Rbm & T & Rc & If \\
\hline 0.147 & 0.156 & 0.218 & 0.073 & 0.121 & 0.113 & 0.073 & 0.057 & 0.023 & 0.020 \\
\hline
\end{tabular}

TOPSIS model is executed using a python library topsispy in Spyder IDE. It requires three input parameters to be executed-vector containing weightage of parameter (Table 3), decision matrix (Table 1), vector containing the preference direction on each criteria (Table 4). Decision matrix shown in Table 1 is prepared for watershed where each row represents a sub-watershed while each column its value of MA. Preference direction is represented by +1 and -1 where +1 is used for positive criteria while -1 for negative criteria. Direction of each criteria is shown in Table 4. Table 4 concludes that each MA except Rc chosen for this study shows the increasing monotonicity or direct relationship with erodibility. Rc is exception because it is a shape parameter as mentioned earlier in this section. This python library will return a tuple with index of most preferred sub-watershed and array containing closest coefficient of all sub-watershed. The sub-watershed with its closest coefficient and rank is shown in Table 5. It is revealed in Table 5 that sub-watershed fid21, fid17, fid7 having closest coefficient $0.794,0.529,0.376$ respectively has given rank from 1 to 3 based on the susceptibility to soil erosion. The closest coefficient is classified into three soil erosion susceptibility classes (Figure 3), namely low (0.147-0.167), medium (0.167-0.326) and high(0.326-0.794) based on geometrical mean method. It is visible from the Figure 3 that highly susceptible zone are well distributed from high elevation to low elevation area. High, medium and low susceptible classes covers the $4.73 \%, 38.79 \%, 56.48 \%$ area of watershed respectively. Finally, the output of TOPSIS-AHP model shows that sub-watershed fid21 should be in highest priority for conservation and management. 
Table 4. Monotonic function and erodibility relation of MAs.

\begin{tabular}{ccc}
\hline MA & Relation with Erodibility & Monotonic Function \\
\hline $\mathrm{Rbm}$ & Directe $(+1)$ & Increasing \\
$\mathrm{Dd}$ & Directe $(+1)$ & Increasing \\
$\mathrm{Fu}$ & Directe $(+1)$ & Increasing \\
$\mathrm{T}$ & Directe $(+1)$ & Increasing \\
$\mathrm{If}$ & Directe $(+1)$ & Increasing \\
$\mathrm{Rc}$ & Inverse $(-1)$ & Decreasing \\
$\mathrm{Rr}$ & Directe $(+1)$ & Increasing \\
$\mathrm{Rn}$ & Directe $(+1)$ & Increasing \\
$\mathrm{Sw}$ & Directe $(+1)$ & Increasing \\
$\mathrm{Lo}$ & Directe $(+1)$ & Increasing \\
\hline
\end{tabular}

Table 5. Erodibility prioritization of sub-watershed based on TOPSIS model.

\begin{tabular}{|c|c|c|c|c|c|c|c|c|c|c|c|}
\hline \multicolumn{12}{|c|}{$\mathbf{a}$} \\
\hline SW & fid0 & fid1 & fid2 & fid3 & fid4 & fid5 & fid6 & fid7 & fid8 & fid9 & fid10 \\
\hline $\mathrm{Ci}+$ & 0.181 & 0.244 & 0.333 & 0.123 & 0.207 & 0.145 & 0.195 & 0.376 & 0.1 & 0.141 & 0.218 \\
\hline Rank & 10 & 5 & 4 & 19 & 8 & 16 & 9 & 3 & 15 & 17 & 7 \\
\hline \multicolumn{12}{|c|}{ b } \\
\hline SW & fic & fid & fid & fid & fid1 & fid & fid & fid & & fid19 & fid21 \\
\hline $\mathrm{Ci}+$ & & 0.1 & 0.1 & 0.1 & 0.22 & 0.1 & 0.5 & 0.1 & & 0.131 & 0.794 \\
\hline Ranl & 2 & 1 & 20 & 1 & 6 & 12 & 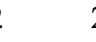 & 1 & & 18 & 1 \\
\hline
\end{tabular}

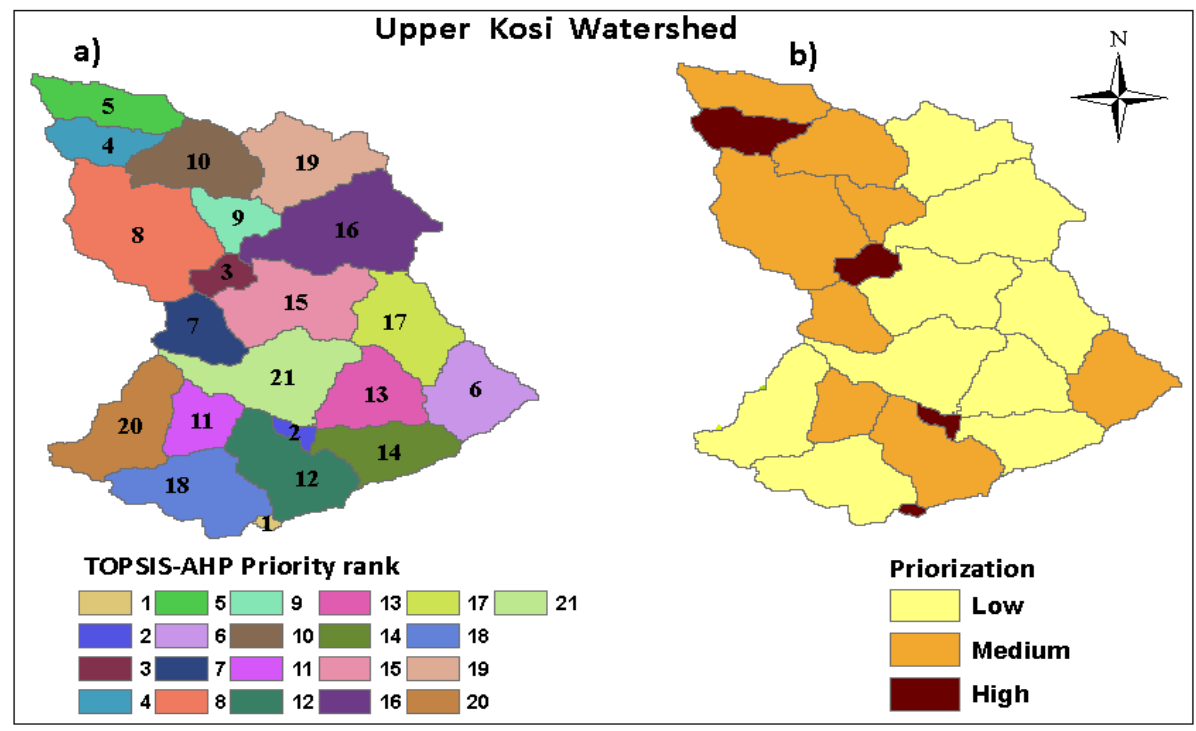

Figure 3. Upper Kosi watersheds: (a) TOPSIS-AHP Priority Rank , (b) Prioritization map.

\section{Conclusions}

TOPSIS-AHP model is utilized for soil erodibility prioritization for Kosi watershed having 5th order perennial river. 21 sub-watersheds were extracted using TanDEM-X 90m DEM. Delineated stream map from DEM shows dendritic drainage pattern in watershed. This is followed by calculation and comparison of $10 \mathrm{MA}$ for each sub-watershed. Exceptionally high value of Sw concludes the risk of high flood peak at the time of rainfall. Other MAs comes under the acceptable range. Sw, Fu, Dd is found to be more impactful as compared to other MAs for soil erosion according to AHP model. 
The result of TOPSIS-AHP ensemble model reveals that $4.73 \%, 38.79 \%, 56.48 \%$ area comes under high, medium and low soil erosion susceptible classes respectively. For the Upper Kosi watershed, sub-watershed having id fid 21 has highest susceptibility to soil erosion whereas fid11 has lowest. So, it is strongly recommended that proper protection measures such as plantation, contour farming etc. should be taken for watershed management of High soil erosion susceptible classes to reduce the erosion, landslide etc. The present study showed that the Remote sensing and GIS technique with MCDM method such as TOPSIS-AHP model can be utilized by decision-makers and planners of soil, water and agriculture department for soil erosion control.

Acknowledgments: We thanks Indian Institute of Remote Sensing for his encouragement and support. The TanDEM-X $90 \mathrm{~m}$ data are copyright of German Aerospace Centre (DLR) and was made available by the EOC Geoservice.

Conflicts of Interest: The authors declare no conflict of interest.

\section{References}

1. Karlen, D.L.; Ditzler, C.A.; Andrews, S.S. Soil quality: Why and how? Geoderma 2003, 114, 145-156.

2. Ribaudo, M. Reducing Soil Erosion: Offsite Benefits; US Department of Agriculture, Economic Research Service: Washington, DC, USA, 1986.

3. Guerra, C.A.; Rosa, I.M.; Valentini, E.; Wolf, F.; Filipponi, F.; Karger, D.N.; Xuan, A.N.; Mathieu, J.; Lavelle, P.; Eisenhauer, N. Global vulnerability of soil ecosystems to erosion. Landsc. Ecol. 2020, 35, 823-842.

4. Seitz, S.; Goebes, P.; Puerta, V.L.; Pereira, E.I.P.; Wittwer, R.; Six, J.; van der Heijden, M.G.; Scholten, T. Conservation tillage and organic farming reduce soil erosion. Agron. Sustain. Dev. 2019, 39, 4.

5. Asfaw, D.; Workineh, G. Quantitative analysis of morphometry on Ribb and Gumara watersheds: Implications for soil and water conservation. Int. Soil Water Conserv. Res. 2019, 7, 150-157.

6. Mokarram, M.; Hojati, M. Morphometric analysis of stream as one of resources for agricultural lands irrigation using high spatial resolution of digital elevation model (DEM). Comput. Electron. Agric. 2017, 142, 190-200.

7. Aher, P.; Adinarayana, J.; Gorantiwar, S. Quantification of morphometric characterization and prioritization for management planning in semi-arid tropics of India: a remote sensing and GIS approach. J. Hydrol. 2014, $511,850-860$.

8. Tripathi, M.; Panda, R.; Raghuwanshi, N. Identification and prioritisation of critical sub-watersheds for soil conservation management using the SWAT model. Biosyst. Eng. 2003, 85, 365-379.

9. Naqvi, H.R.; Athick, A.M.A.; Ganaie, H.A.; Siddiqui, M.A. Soil erosion planning using sediment yield index method in the Nun Nadi watershed, India. Int. Soil Water Conserv. Res. 2015, 3, 86-96.

10. Jaiswal, R.; Thomas, T.; Galkate, R.; Ghosh, N.; Singh, S. Watershed prioritization using Saaty's AHP based decision support for soil conservation measures. Water Resour. Manag. 2014, 28, 475-494.

11. Rahaman, S.A.; Ajeez, S.A.; Aruchamy, S.; Jegankumar, R. Prioritization of sub watershed based on morphometric characteristics using fuzzy analytical hierarchy process and geographical information system-A study of Kallar Watershed, Tamil Nadu. Aquat. Procedia 2015, 4, 1322-1330.

12. Aouragh, M.H.; Essahlaoui, A. A TOPSIS approach-based morphometric analysis for sub-watersheds prioritization of high Oum Er-Rbia basin, Morocco. Spat. Inf. Res. 2018, 26, 187-202.

13. Ganie, P.A.; Posti, R.; Kumar, P.; Singh, A. Morphometric analysis of a Kosi River Basin, Uttarakhand using geographical information system. Int. J. Multidiscip. Curr. Res. 2016, 4, 1190-1200.

14. Grohmann, C.H. Evaluation of TanDEM-X DEMs on selected Brazilian sites: Comparison with SRTM, ASTER GDEM and ALOS AW3D30. Remote. Sens. Environ. 2018, 212, 121-133.

15. Sadhasivam, N.; Bhardwaj, A.; Pourghasemi, H.R.; Kamaraj, N.P. Morphometric attributes-based soil erosion susceptibility mapping in Dnyanganga watershed of India using individual and ensemble models. Environ. Earth Sci. 2020, 79, 1-28.

16. Li, Z. Watershed modeling using arc hydro based on DEMs: a case study in Jackpine watershed. Environ. Syst. Res. 2014, 3, 11.

17. Mardani, A.; Jusoh, A.; Nor, K.; Khalifah, Z.; Zakwan, N.; Valipour, A. Multiple criteria decision-making techniques and their applications-a review of the literature from 2000 to 2014. Econ. Res. Ekon. Istraživanja 2015, 28, 516-571. 
18. Kumar, A.; Sah, B.; Singh, A.R.; Deng, Y.; He, X.; Kumar, P.; Bansal, R. A review of multi criteria decision making (MCDM) towards sustainable renewable energy development. Renew. Sustain. Energy Rev. 2017, 69, 596-609.

19. Saaty, T.L. Decision making—the analytic hierarchy and network processes (AHP/ANP). J. Syst. Sci. Syst. Eng. 2004, 13, 1-35.

20. Harker, P.T.; Vargas, L.G. The theory of ratio scale estimation: Saaty's analytic hierarchy process. Manag. Sci. 1987, 33, 1383-1403.

21. Yoon, K.; Hwang, C.L. TOPSIS (Technique for Order Preference by Similarity to Ideal solution)—A Multiple Attribute Decision Making, w: Multiple Attribute Decision Making-Methods and Applications, a State-of-the-At Survey. Springer: Berlin, Germany, 1981.

22. Krieger, G.; Moreira, A.; Fiedler, H.; Hajnsek, I.; Werner, M.; Younis, M.; Zink, M. TanDEM-X: A Satellite Formation for High-Resolution SAR Interferometry. IEEE Trans. Geosci. Remote. Sens. 2007, 45, 3317-3341.

23. Amiri, M.; Pourghasemi, H.R.; Arabameri, A.; Vazirzadeh, A.; Yousefi, H.; Kafaei, S. Prioritization of flood inundation of Maharloo Watershed in iran using morphometric parameters analysis and TOPSIS MCDM model. In Spatial Modeling in GIS and $R$ for Earth and Environmental Sciences; Elsevier: Amsterdam, The Netherlands, 2019; pp. 371-390.

Publisher's Note: MDPI stays neutral with regard to jurisdictional claims in published maps and institutional affiliations.

(C) 2020 by the authors. Licensee MDPI, Basel, Switzerland. This article is an open access article distributed under the terms and conditions of the Creative Commons Attribution (CC BY) license (http://creativecommons.org/licenses/by/4.0/). 